\title{
Importância da orientação nutricional e do teor de fibras da dieta no controle glicêmico de pacientes diabéticos tipo 2 sob intervenção educacional intensiva
}

\author{
Importance of nutritional counseling and dietary \\ fiber content on glycemic control in type 2 diabetic \\ patients under intensive educational intervention
}

Fernanda Sanches Carvalho', Augusto Pimazoni Netto', Patrícia Zach', Anita Sachs², Maria Teresa Zanella'

1 Departamento de Medicina, Endocrinologia, Escola Paulista de Medicina, Universidade Federal de São Paulo (Unifesp/ EPM), São Paulo, SP, Brasil 2 Departamento de Medicina Preventiva, Unifesp/EPM São Paulo, SP, Brasil
Correspondência para: Fernanda Sanches Carvalho Rua Pedro de Paula Leite, 468, sala 2

13300-520 - Itu, SP, Brasil fe_sanchesc@hotmail.com

Recebido em 17/Maio/2011 Aceito em 17/Fev/2012

\section{RESUMO}

Objetivo: Avaliar a orientação nutricional dentro de um conjunto de intervenções multidisciplinares. Sujeitos e métodos: Quarenta e sete pacientes diabéticos tipo 2, hiperglicêmicos, tratados de forma convencional $(n=19)$ ou intensiva $(n=28)$ e avaliados pela glicemia média semanal (GMS) no início e após 6 semanas. Resultados: GI reduziu o consumo de calorias $(p=0,001)$, carboidratos $(p=0,004)$, lipídios $(p=0,001)$ e aumentou o de fibras, enquanto o $G C$ reduziu o consumo de fibras $(p=0,018)$. Controle glicêmico (GMS $\leq 150 \mathrm{mg} / \mathrm{dL}$ ) ocorreu em $75 \%$ do $\mathrm{Gl}$ e, em $31,6 \%$ do GC $(p=0,003)$, houve correlação negativa entre as variações do consumo de fibras e a GMS $(r=-0,309 ; p=0,035)$. Os resultados mantiveram-se por 12 semanas. Conclusão: A intervenção educacional intensiva de curto prazo mostrou-se mais eficaz que o tratamento convencional para a obtenção do controle glicêmico. Nossos resultados ainda indicam que um consumo mais adequado de fibras na alimentação contribui para a obtenção de um melhor controle da glicemia. Arq Bras Endocrinol Metab. 2012;56(2):110-9

Descritores

Orientação nutricional; diabetes tipo 2; controle glicêmico; glicemia média semanal

\section{ABSTRACT}

Objective: To evaluate the importance of nutritional counseling within a set of multidisciplinary interventions. Subjects and methods: Forty-seven patients with type 2 diabetes and hyperglycemia (A1C $\geq 8 \%$ ), treated conventionally $(n=19, G C)$ or intensively in six weekly visits $(n=$ $28, \mathrm{Gl}$ ) were analyzed. We evaluated mean weekly blood glucose (MWG) at baseline and after 6 weeks in both groups. Results: GI reduced caloric $(p=0.001)$, carbohydrate $(p=0.004)$, and fat $(p=0.001)$ intake, and increased fiber consumption, while GC reduced fiber intake $(p=0.018)$. Glycemic control (MWG $\leq 150 \mathrm{mg} / \mathrm{dL}$ ) occurred in $75 \%$ of Gl patients and in $31.6 \%$ of CG patients $(p=0.003)$, with negative correlation between changes in fiber intake and MWG values $(r=-$ $0.309 ; P=0.035$ ). Results were maintained after 12 weeks. Conclusion: Educational short-term intensive intervention was more effective than conventional treatment to achieve glycemic control. Our results also indicate that a more appropriate fiber content in the diet contributes for better blood glucose control in these patients. Arq Bras Endocrinol Metab. 2012;56(2):110-9

\section{Keywords}

Nutritional counseling; type 2 diabetes; glycemic control; mean weekly blood glucose 


\section{INTRODUÇÃO}

O diabetes melito (DM) é uma doença de etiologia múltipla, decorrente da falta de insulina bem como da incapacidade desta de exercer adequadamente seus efeitos, resultando em resistência à insulina. Caracteriza-se pela presença de hiperglicemia crônica, frequentemente acompanhada de dislipidemia, obesidade abdominal, hipertensão arterial e disfunção endotelial. $\mathrm{O}$ conjunto desses fatores pode elevar o risco de desenvolver outras comorbidades, tais como doenças cardiovasculares (1-3). Atualmente no Brasil observa-se aumento na prevalência de DM tipo 2 (DM2) devido ao envelhecimento populacional, à crescente prevalência de obesidade, a fatores relacionados ao estilo de vida, além de modificações no consumo alimentar. Nota-se baixa frequência de alimentos ricos em fibras, tais como frutas e hortaliças, aumento da proporção de gorduras saturadas e açúcares da dieta (2-4).

Autores têm demonstrado que mudanças na alimentação em pacientes portadores de DM2, tais como consumo de alimentos com baixo índice glicêmico e ricos em fibras alimentares, induzem menor aumento nos níveis séricos de glicose e insulina no período pós-prandial (5). O consenso publicado pela American Diabetes Association (ADA) e European Association for the Study of Diabetes (EASD) sinaliza a mudança de estilo de vida de suma importância no tratamento do DM (6). Sendo assim, as orientações nutricionais, associadas às mudanças de estilo de vida, são consideradas essenciais para o controle do DM (7). Porém, a adesão às recomendações nutricionais nem sempre é satisfatória (8). Com base nessa afirmação, torna-se necessário conscientizar o paciente sobre a importância da adesão ao tratamento, que resultará em melhor controle da doença (9).

O Grupo de Educação e Controle do Diabetes (GECD) do Hospital do Rim e Hipertensão/Universidade Federal de São Paulo (Unifesp) propõe intervenção multidisciplinar e uma nova abordagem para o tratamento do diabetes, utilizando o conceito da glicemia média semanal (GMS) e variabilidade glicêmica (VG), cuja essência é direcionar o tratamento objetivando atingir o controle glicêmico em um período de seis semanas. Trata-se de um método baseado na automonitorização domiciliar para avaliação do controle glicêmico do paciente. Os resultados são avaliados semanalmente para que se possa adequar o tratamento dietético e farmacológico a condições de controle de cada paciente.
Assim, este estudo teve por objetivo avaliar a importância da orientação nutricional dentro de um conjunto de intervenções educacionais de curto prazo para a obtenção de melhor controle da glicemia em pacientes diabéticos tipo 2 .

\section{SUJEITOS E MÉTODOS}

\section{Paciente e local de estudo}

$\mathrm{O}$ atual estudo faz parte do projeto intitulado: "A glicemia média semanal como método de avaliação em tempo quase real do controle glicêmico e da adequação da conduta terapêutica no diabetes tipo 2: um estudo prova de conceito" e teve por objetivo avaliar a importância da orientação nutricional na obtenção do controle glicêmico em pacientes com DM2. Trata-se de um estudo prospectivo, controlado, aberto, com duração de doze semanas em indivíduos $(\mathrm{n}=47)$ com DM2, de ambos os sexos, com idade mínima de 30 anos e máxima de 75 anos, em tratamento com antidiabéticos orais ou insulina há pelo menos três meses, dose estável da medicação anti-hipertensiva e/ou hipolipemiante quando houvesse indicação e apresentando níveis de hemoglobina glicada (AlC) $\geq 8 \%$. Os indivíduos foram recrutados no Ambulatório de Hipertensão e Diabetes do Centro Integrado de Hipertensão e Metabologia Cardiovascular do Hospital do Rim e Hipertensão (CIHMCV), Universidade Federal de São Paulo (Unifesp). O estudo foi aprovado pelo Comitê de Ética em Pesquisa da Unifesp (CEP 1667/08 em 30 de janeiro de 2009). Todos os participantes assinaram o termo de consentimento livre e esclarecido.

Foram excluídos da amostra os pacientes portadores de doenças graves, tais como insuficiência hepática, insuficiência cardíaca congestiva (graus 3 e 4), insuficiência renal com valores de creatinina superiores a $3,0 \mathrm{mg} / \mathrm{dL}$, hipertensão arterial secundária ou com valores da pressão arterial sistólica $\geq 180 \mathrm{mmHg}$ ou diastólica $\geq 110 \mathrm{mmHg}$, deficiências psiquiátricas graves, ocorrência de evento cardiovascular dentro de um período prévio inferior a três meses ou, ainda, aqueles que apresentassem quaisquer condições que interferissem no resultado ou na condução do estudo, como dependência química, recusa à terapêutica com insulina, dificuldade de comparecer às consultas, dificuldade de compreender as orientações, uso de medicamentos (que não antidiabéticos) que pudessem interferir na resistência à insulina, ou cirurgia bariátrica anterior. 
Os pacientes incluídos foram divididos aleatoriamente em dois grupos: grupo convencional e grupo intervenção. O grupo intervenção (GI) foi tratado de forma intensiva por uma equipe multiprofissional constituída por um médico, nutricionistas, educador em diabetes, enfermeiras, psicólogas e educador físico, mediante visitas semanais por um período de seis semanas consecutivas e avaliado após seis e 12 semanas de tratamento, enquanto o grupo convencional (GC) foi tratado e recebeu orientação apenas de um médico, tendo sido avaliado seis e 12 semanas após o início do estudo.

\section{Protocolo do estudo}

No início do estudo (visita -1), com os medicamentos em uso mantidos, todos os indivíduos que obedeceram aos critérios de inclusão e exclusão receberam um monitor de glicemia (glicosímetro) para uso individual e tiras reagentes para a realização de três perfis glicêmicos diários constituídos cada um por sete pontos, num total de 21 testes por semana. Os testes eram realizados em jejum, 2 horas após o café da manhã, antes do almoço, 2 horas após o almoço, antes do jantar, 2 horas após o jantar e ao deitar-se. Os pacientes insulinizados realizavam um teste adicional durante a madrugada (entre 2 e 4 horas).

Após a visita inicial (-1) iniciou-se a semana denominada semana 0 . Nesse período, os pacientes dos dois grupos eram submetidos à coleta de sangue em jejum para determinação dos valores da AlC e da glicemia de jejum, realizavam o controle glicêmico domiciliar e preenchiam o inquérito alimentar. Ao final da semana zero (visita 0 ), os pacientes dos dois grupos eram submetidos ao exame antropométrico (peso, estatura e circunferência da cintura) e entregavam o registro alimentar, sendo este conferido por um nutricionista treinado. Os glicosímetros com os registros das glicemias eram trazidos pelos pacientes para análise conjunta da equipe multidisciplinar, que avaliava os perfis diários da glicemia e realizava os cálculos das glicemias médias semanais (GMS) e dos respectivos desvios-padrão (DP). Nessa ocasião, a fim de avaliar o consumo alimentar em ambos os grupos (intervenção e controle), os pacientes recebiam orientações para preenchimento do inquérito alimentar denominado "Registro alimentar de 3 dias", sendo estes dias consecutivos. Com base no perfil glicêmico dessa semana, era iniciado o tratamento do grupo GI, enquanto a AlC e a glicemia de jejum eram os parâmetros utilizados para início do tratamento do GC.
A partir da visita 0 , o grupo intervenção realizava visitas semanais por um período de 6 semanas, durante o qual recebia orientações da equipe multidisciplinar. Neste grupo, ajustes no tratamento farmacológico e não farmacológico do diabetes eram realizados semanalmente, baseados nos resultados do controle domiciliar das glicemias capilares realizadas nos três dias que precediam a visita à equipe multiprofissional. Os pacientes do grupo convencional eram avaliados e orientados pelo médico responsável no início do estudo, com base nos valores da glicemia de jejum e da hemoglobina glicada, e eram novamente avaliados ao final da semana 6 , sendo que durante esta última semana faziam também o controle domiciliar da glicemia. Assim sendo, como era de se esperar, os ajustes semanais na medicação do grupo GI fizeram com que, ao final da semana 6, os dois grupos, que eram semelhantes quanto ao tratamento farmacológico inicial, passassem a diferir quanto ao consumo de doses e número de medicamentos antidiabéticos. Na semana 6 , os dois grupos eram novamente submetidos a coletas de sangue em jejum para avaliação da glicemia e AlC, determinavam novamente o perfil da glicemia domiciliar e preenchiam o registro alimentar de três dias. Ao final da semana 6, (visita 6) eram repetidos os procedimentos da visita 0 e igualmente feitos os ajustes na terapêutica dos pacientes dos dois grupos. A partir dessa semana, os pacientes eram orientados a manter o tratamento até a semana 12, sendo que, nessa semana, repetiam-se todos os procedimentos descritos para a semana 6 e visita 6 .

Foi considerado bom controle glicêmico a média da glicemia capilar semanal menor ou igual a $150 \mathrm{mg} / \mathrm{dL}$, após seis semanas de tratamento.

Com relação à atenção nutricional, os pacientes do grupo intervenção receberam orientação nutricional individualizada, com base na anamnese alimentar, no inquérito alimentar e no gasto energético estimado, respeitando o histórico pessoal de doenças e hábitos alimentares, visando manter ou atingir o peso adequado, bem como prevenir e controlar complicações e outros problemas metabólicos associados. Os indivíduos com excesso de peso receberam plano alimentar visando uma perda de peso modesta $(5 \%)$, com redução entre 500 a $1.000 \mathrm{kcal}$, a partir do gasto energético estimado na avaliação nutricional inicial $(7,10)$. Dessa forma, cada participante desse grupo foi atendido, semanalmente, por nutricionistas treinados, durante o período de intervenção. As orientações nutricionais foram baseadas nas recomendações da Associação Americana 
de Diabetes (7) e da Sociedade Brasileira de Diabetes (2). A recomendação de macronutrientes proposta foi de $15 \%$ a $20 \%$ da ingestão energética total de proteínas, aproximadamente $30 \%$ de lipídios, sendo gordura saturada $\leq 7 \%$ e colesterol $<200 \mathrm{mg}$, carboidratos 45\%-65\%, perfazendo $60 \%$ a $70 \%$ da ingestão energética associado à gordura monoinsaturada. Com relação às fibras, o preconizado foi uma ingestão mínima de $20 \mathrm{~g}$ ao dia, sob a forma de hortaliças, leguminosas, grãos integrais e frutas $(7,2)$ ou um consumo mínimo de $14 \mathrm{~g}$ de fibra para $1.000 \mathrm{kcal} /$ dia ingeridas (11). Objetivando atingir essas recomendações, as orientações visavam inicialmente à mudança do estilo de vida.

$\mathrm{Na}$ visita 0 , após o inquérito alimentar, os pacientes receberam orientações sobre os "Dez passos para alimentação saudável dos diabéticos”, preconizados na publicação do Guia Alimentar para pessoas com Diabetes Tipo 2, do Ministério da Saúde (12). Na visita 1, foram entregues o plano alimentar individualizado e uma ilustração do "Prato Colorido - Alimentação Saudável" da campanha do Conselho Federal de Nutricionista (13). Nas visitas seguintes, os nutricionistas checavam as dificuldades e esclareciam possíveis dúvidas. Com relação ao grupo convencional, o médico responsável fornecia informações básicas sobre a alimentação para paciente diabético. Dessa forma, eles foram orientados a realizar fracionamento das refeições, inclusão de hortaliças (cruas e cozidas), frutas ( 3 porções ao dia) e inclusão de carboidratos integrais, quando possível. Vale ressaltar que não foi entregue impresso com uma "dieta padrão" a esses pacientes.

Com relação à avaliação do consumo alimentar, os alimentos registrados em medidas caseiras pelos pacientes eram codificados pelo nutricionista responsável, ou seja, convertidos em gramas $(14,15)$.

Para a determinação da composição química dos alimentos, em relação aos de macronutrientes e conteúdo de fibras, utilizou-se o programa Nut Win ${ }^{\circledR}(16)$. Os alimentos ou as preparações não existentes no programa foram acrescentados, utilizando as informações contidas nas seguintes tabelas de composição química dos alimentos: Tabela de Composição de Alimentos versão 2 - TACO2 (17), Tabela de Composição de Química dos Alimentos - USDA (18) e Tabela Brasileira de Composição dos Alimentos - TBCA USP (19).

Os níveis da glicemia capilar eram determinados por meio de um glicosímetro da marca Accu-Chek ${ }^{\circledR}$ Performa (Roche Diagnostics). Os cálculos das glicemias médias semanais (GMS) e dos respectivos desvios-padrão foram realizados eletronicamente pelo software Accu-Chek ${ }^{\circledR} 360^{\circ}$ - Diabetes Management System (Roche Diagnostics).

Os níveis plasmáticos de hemoglobina glicada (AlC) foram avaliados com a utilização do método de inibição de imunoaglutinação em látex com o auxílio do analisador DCA $2000^{\circledR}$ (Siemens Medical Solutions Diagnostics) devidamente certificado pelo National Glycohemoglobin Standardization Program (NGSP).

O peso corporal foi avaliado, em quilogramas, utilizando-se balança antropométrica digital $\left(\right.$ Filizola $\left.^{\circledR}\right)$ do tipo plataforma com capacidade de $180 \mathrm{~kg}$ e precisão de $100 \mathrm{~g}$, posicionada sob superfície plana. Os indivíduos eram pesados descalços, utilizando roupas leves (20). A estatura foi aferida por meio de estadiômetro fixo da mesma balança e os participantes foram orientados a permanecer descalços, na posição ereta com os braços pendentes ao longo do corpo (21). As medidas de peso e estatura foram utilizadas para determinar o índice de massa corporal (IMC), que é calculado dividindo-se a medida do peso $(\mathrm{kg})$ pela medida da estatura (m) elevada ao quadrado e classificado de acordo com o proposto pela Organização Mundial da Saúde (22).

A circunferência de cintura foi medida com fita métrica inextensível e flexível, com aproximação em $0,1 \mathrm{~cm}$, com o indivíduo na posição ereta, abdômen relaxado, braços ao lado do corpo e os pés juntos, sendo a medida realizada no ponto médio entre a última costela e a crista ilíaca (22).

\section{Análise estatística}

Os dados foram analisados no programa estatístico SPSS versão 17. O teste de Kolmogorov Smirnov foi utilizado para verificar a normalidade das variáveis. Para comparar os valores das variáveis obtidas ao final das semanas 0 e 6, utilizou-se o teste $t$ de Student para médias dependentes ( $t$ pareado). Ao comparar as variáveis dos dois grupos no momento inicial do estudo, usou-se o teste $t$ de Student para médias independentes $(t$ não pareado). $O$ teste do $\chi^{2}$ foi utilizado para testar a associação entre bom controle glicêmico e programa de tratamento (convencional ou intensivo). Para avaliar a correlação entre as variáveis e o controle glicêmico, medido por meio da GMS, utilizou-se o teste de correlação de Pearson. Os resultados foram expressos em média e desvio-padrão. Para todos os testes, adotou-se o nível de significância inferior a 0,05 ou $5 \%$. 


\section{RESULTADOS}

Dos 61 pacientes selecionados para participar do GECD, incluídos de forma aleatória em grupo controle (29 indivíduos) e grupo intervenção (32 indivíduos), foi possível analisar para este estudo 47 indivíduos. Excluíram-se os pacientes com dados incompletos do inquérito alimentar. As características dos pacientes estudados em cada grupo, no momento inicial do estudo, estão demonstradas na tabela 1 . Não houve diferença estatisticamente significativa na idade observada entre os grupos $(\mathrm{p}=0,322)$. Em relação ao sexo, notou-se um predomínio semelhante do sexo feminino em ambos os grupos. O tempo de diagnóstico da doença foi também semelhante entre os grupos. Com relação ao tratamento inicial, observou-se um número maior de pacientes em uso de insulina concomitante à terapia com antidiabéticos orais no grupo intervenção e à monoterapia com insulina no grupo convencional. Quanto à escolaridade, a maior parte dos indivíduos apresentava mais de oito anos de estudo.

Com relação ao consumo alimentar, no momento inicial do estudo, os grupos eram semelhantes, porém houve uma diferença estatisticamente significativa no tocante ao consumo de fibra ajustado para o conteúdo calórico da dieta (grama/1.000 kcal), sendo esse consumo maior no grupo convencional do que no grupo intervenção. Quanto à distribuição de macronutrientes na dieta, em relação ao percentual do valor calórico total (VCT), a ingestão média de carboidratos e proteínas de ambos os grupos estava de acordo com o preconizado para pacientes diabéticos, porém o consumo de lipídios estava um pouco acima do ideal $(25 \%$ a $35 \%$ do VCT) $(2,7)$.

Tabela 1. Características basais (visita 0) dos grupos intervenção e convencional

\begin{tabular}{|c|c|c|c|}
\hline & $\begin{array}{c}\text { Grupo intervenção } \\
(\mathrm{n}=\mathbf{2 8}) \\
\text { Semana } 0\end{array}$ & $\begin{array}{c}\text { Grupo convencional } \\
(n=19) \\
\text { Semana } 0\end{array}$ & Valor $\mathbf{P}$ \\
\hline Idade (anos) & $55,14(8,68)$ & $57,74(8,77)$ & 0,322 \\
\hline \multicolumn{4}{|l|}{ Sexo } \\
\hline Masculino & $7(25 \%)$ & $4(21,1 \%)$ & 0,754 \\
\hline Feminino & $21(75 \%)$ & $15(78,9 \%)$ & \\
\hline Tempo de diagnóstico do diabetes (anos) & $11,54(7,82)$ & $13,11(7,29)$ & 0,492 \\
\hline \multicolumn{4}{|l|}{ Tratamento do diabetes (DM2) } \\
\hline Antidiabético oral (ADO) & $7(25 \%)$ & $6(31,6 \%)$ & \\
\hline Insulina e ADO & $16(57,1 \%)$ & $3(15,8 \%)$ & $0,01^{*}$ \\
\hline Monoterapia com insulina & $5(17,9 \%)$ & $10(52,6 \%)$ & \\
\hline \multicolumn{4}{|l|}{ Escolaridade } \\
\hline$<4$ anos & $2(7,1 \%)$ & $4(21,1 \%)$ & \\
\hline $4-8$ anos & $9(32,1 \%)$ & $7(36,8 \%)$ & 0,283 \\
\hline$>8$ anos & $17(60,7 \%)$ & $8(42,1 \%)$ & \\
\hline \multicolumn{4}{|l|}{ Características clínicas } \\
\hline Hemoglobina glicada (A1C\%) & $10,08(1,33)$ & $9,87(1,28)$ & 0,582 \\
\hline GMS & $214,82(49,14)$ & $201,54(44,78)$ & 0,351 \\
\hline Variabilidade glicêmica (VG) ou dp & $68,64(26,03)$ & $67,12(24,71)$ & 0,842 \\
\hline IMC $\left(\mathrm{kg} / \mathrm{m}^{2}\right)$ & $32,01(6,42)$ & $30,84(5,26)$ & 0,514 \\
\hline Circunferência da cintura (cm) & $105,84(15,52)$ & $99,96(10,81)$ & 0,160 \\
\hline \multicolumn{4}{|l|}{ Consumo alimentar } \\
\hline Caloria (kcal) & $1.744,34(484,56)$ & $1.588,93(409,36)$ & 0,258 \\
\hline Carboidrato (g) & $200,74(63,10)$ & $179,56(48,39)$ & 0,223 \\
\hline Carboidrato (\% vct) & $45,99(7,03)$ & $45,47(8,11)$ & 0,817 \\
\hline Proteína (g) & $79,45(21,98)$ & $80,92(42,49)$ & 0,877 \\
\hline Proteína (\%vct) & $18,71(4,57)$ & $19,76(6,19)$ & 0,508 \\
\hline Lipídios (g) & $69,48(24,78)$ & $61,76(16,59)$ & 0,241 \\
\hline Lipídios (\%vct) & $35,51(4,78)$ & $35,32(5,41)$ & 0,902 \\
\hline Fibra (g) & $18,91(7,27)$ & $21,91(9,46)$ & 0,225 \\
\hline Fibra ajustada (g/1.000 kcal) & $11,05(3,26)$ & $13,61(4,34)$ & $0,036^{*}$ \\
\hline
\end{tabular}

VCT: valor calórico total. * $p<0,05$. 
A tabela 2 mostra os valores das variáveis obtidos nas semanas 0 e 6 em cada grupo. Verificou-se uma melhora nos parâmetros glicêmicos em ambos os grupos, tais como AlC, GMS e variabilidade glicêmica. No entanto, houve associação entre o tratamento intensivo e o controle glicêmico, observando-se um maior número de pacientes no grupo intensivo que atingiu a média da glicemia capilar semanal menor ou igual a $150 \mathrm{mg} /$ $\mathrm{dL}$ em um período de seis semanas ( $75 \%$ vs. $31,6 \% ; \mathrm{p}=$ $0,003)$. Comparando-se os valores obtidos nos grupos, observou-se uma diferença estatisticamente significativa na GMS da semana $6(137 \mathrm{mg} / \mathrm{dL}$ vs. $176 \mathrm{mg} / \mathrm{dL}$; $\mathrm{p}=0,000) \mathrm{e}$, apesar de o valor da hemoglobina glicada ser menor no grupo intervenção, essa diferença não foi significativa $(\mathrm{p}=0,093)$.

Quanto ao consumo alimentar, ao se analisar o grupo intervenção, observou-se uma redução do valor calórico $(\mathrm{p}=0,001)$. Apesar dessa redução, não foi observada alteração estatisticamente significativa do valor do IMC ( $p=0,649)$, embora se observasse uma redução da circunferência da cintura $(\mathrm{p}=0,022)$. Notou-se diminuição do consumo de carboidratos, em gramas ( $\mathrm{p}$ $=0,004$ ), porém sem diferença estatisticamente significativa em relação ao percentual do valor calórico total (VCT) para esse nutriente $(\mathrm{p}=0,166)$. Além disso, observou-se diminuição da quantidade de lipídios consumida, tanto em gramas como em porcentagem referen- te ao VCT. Dessa forma, a redução na ingestão calórica se deveu à diminuição da ingestão lipídica. Com relação ao consumo de fibras, foi observado aumento da fibra ajustada para calorias (grama/1.000 kcal).

Ao analisarmos o grupo convencional, não foi verificada diferença estatisticamente significativa no consumo dos macronutrientes (Tabela 2). Com relação ao consumo de fibra, observou-se diminuição tanto da fibra total quanto da fibra ajustada para calorias $(\mathrm{p}=$ 0,018; $\mathrm{p}=0,045)$.

Analisando qualitativamente o consumo de frutas do grupo convencional, observou-se redução de $23 \%$ das porções consumidas, comparando a semana $0 \mathrm{com}$ a semana 6 .

Após um período de mais seis semanas de intervalo, os mesmos parâmetros foram reavaliados em ambos os grupos (semana 12) (Tabela 2).

Ao se analisar as variações no consumo alimentar dos diversos nutrientes com as variações no perfil glicêmico, na semana 6 , nos dois grupos analisados em conjunto ( $n=47)$, não foram observadas correlações entre as variações no consumo de calorias e a GMS ( $\mathrm{p}$ $=0,552)$, de carboidratos e GMS $(\mathrm{p}=0,922)$, de proteínas e GMS ( $\mathrm{p}=0,722)$, ou de lipídios e GMS ( $\mathrm{p}=$ $0,296)$. Todavia, observou-se uma correlação negativa entre as variações no consumo de fibras e na GMS na semana $6(\mathrm{r}=-0,309 ; \mathrm{p}=0,035)$ ilustrada na figura 1 .

Tabela 2. Características das variáveis na semana 0, semana 6 e 12 (média e dp)

\begin{tabular}{|c|c|c|c|c|c|c|c|c|}
\hline \multirow[b]{2}{*}{ Variáveis } & \multicolumn{4}{|c|}{ Grupo intervenção $(n=28)$} & \multicolumn{4}{|c|}{ Grupo convencional $(n=19)$} \\
\hline & Semana 0 & Semana 6 & Semana 12 & $\begin{array}{c}\text { Valor } \\
P\end{array}$ & Semana 0 & Semana 6 & Semana 12 & $\begin{array}{c}\text { Valor } \\
\mathbf{P}\end{array}$ \\
\hline \multicolumn{9}{|l|}{ Características clínicas } \\
\hline $\mathrm{A} 1 \mathrm{C}(\%)$ & $10,08(1,33)$ & $8,4(1,22)$ & $7,81(1,24)$ & $0,000^{*}$ & $9,87(1,28)$ & $9,07(1,44)$ & $8,77(1,32)$ & $0,001^{*}$ \\
\hline GMS (MG/dL) & $214,82(49,14)$ & $137,71(25,66)$ & $148,75(41,78)$ & $0,000^{\star}$ & $201,54(44,78)$ & $176,78(37,52)$ & $169,24(49,35)$ & $0,030^{*}$ \\
\hline VG ou dp & $68,64(26,03)$ & $53,07(22,77)$ & $52,49(20,73)$ & $0,000^{*}$ & $67,12(24,71)$ & $60,43(23,67)$ & $58,53(23,55)$ & 0,144 \\
\hline IMC $\left(\mathrm{kg} / \mathrm{m}^{2}\right)$ & $32,05(6,42)$ & $31,94(6,12)$ & $32,59(5,72)$ & 0,649 & $30,84(5,26)$ & $30,96(5,38)$ & $31,05(5,47)$ & 0,417 \\
\hline Circunferência da cintura (cm) & $105,84(15,52)$ & $104,77(14,60)$ & $107,09(12,74)$ & $0,022^{\star}$ & $99,96(10,81)$ & $100,03(11,51)$ & $100,01(11,75)$ & 0,873 \\
\hline \multicolumn{9}{|l|}{ Consumo alimentar } \\
\hline Caloria (kcal) & $1744,34(484,56)$ & $1520,01(338,01)$ & $1553,15(386,72)$ & $0,001^{*}$ & $1588,94(409,36)$ & $1568,41(369,44)$ & $1602,13(352,43)$ & 0,700 \\
\hline Carboidrato (g) & $200,74(63,10)$ & $179,28(41,57)$ & $179,16(43,44)$ & $0,004^{\star}$ & $179,56(48,39)$ & $177,21(37,32)$ & $186,97(43,20)$ & 0,804 \\
\hline Carboidrato (\% vct) & $45,99(7,03)$ & $47,57(6,22)$ & $46,41(5,43)$ & 0,166 & $45,47(8,11)$ & $46,19(7,17)$ & $46,24(6,86)$ & 0,736 \\
\hline Proteína (g) & $79,45(21,98)$ & $75,80(24,23)$ & $77,76(19,77)$ & 0,447 & $80,92(42,49)$ & $79,08(31,14)$ & $75,61(26,28)$ & 0,886 \\
\hline Proteína (\%vct) & $18,71(4,57)$ & $20,09(4,11)$ & $20,27(3,79)$ & 0,191 & $19,76(6,19)$ & $20,00(4,78)$ & $18,59(4,05)$ & 0,867 \\
\hline Lipídios (g) & $69,48(24,78)$ & $56,75(16,37)$ & $58,98(19,50)$ & $0,001^{*}$ & $61,76(16,59)$ & $60,90(19,44)$ & $60,67(17,26)$ & 0,772 \\
\hline Lipídios (\%vct) & $35,51(4,78)$ & $33,23(4,48)$ & $33,72(4,26)$ & $0,015^{\star}$ & $35,32(5,41)$ & $34,43(4,38)$ & $34,70(5,61)$ & 0,475 \\
\hline Fibra (g) & $18,91(7,27)$ & $20,29(6,61)$ & $19,87(6,79)$ & 0,196 & $21,91(9,46)$ & $17,45(6,18)$ & $20,41(8,13)$ & $0,018^{*}$ \\
\hline Fibra ajustada (g/1.000 kcal) & $11,05(3,26)$ & $13,29(3,42)$ & $12,87(3,43)$ & $0,002^{\star}$ & $13,61(4,34)$ & $11,50(3,68)$ & $12,70(3,63)$ & $0,045^{\star}$ \\
\hline
\end{tabular}

Vct: valor calórico total. * $p<0,05$ : comparação semana 0 e semana 6. 


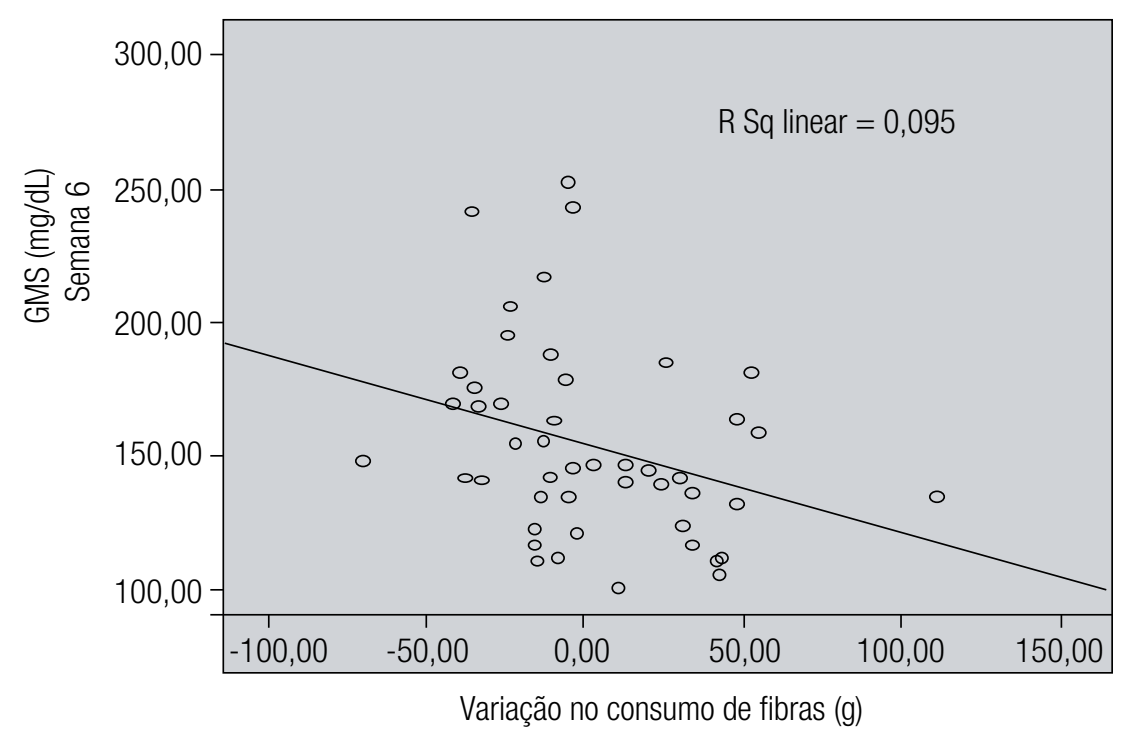

Figura 1. Correlação entre as variações observadas no consumo de fibras e as alterações na GMS.

\section{DISCUSSÃO}

O GECD propõe uma intervenção com equipe multidisciplinar e uma abordagem diferenciada para o tratamento do diabetes, visando controlar a glicemia e melhorar as condições de vida desses pacientes, pelo conceito da glicemia média semanal. Ou seja, método baseado na automonitorização domiciliar para avaliar o controle glicêmico por meio de três perfis glicêmicos semanais. Sabe-se que o uso do perfil glicêmico na prática clínica é de grande valia para a avaliação em curto prazo do controle glicêmico, proporcionando uma conduta terapêutica mais eficaz. A utilização esporádica e não estruturada de testes de glicemia capilar não fornece os elementos necessários para a avaliação completa do estado glicêmico (23). Além do mais, a automonitorização é uma ferramenta prática para ajudar o paciente diabético a compreender melhor a sua doença e as metas do tratamento $(24,25)$.

Os pacientes incluídos neste estudo apresentavam níveis basais de controle glicêmico muito além do recomendado (AlC > 7\%), portanto apresentavam um alto risco de desenvolver complicações micro e macrovasculares decorrentes do DM (2). O tratamento intensivo em comparação ao tratamento convencional mostrou-se mais eficaz para o controle glicêmico, uma vez que, em um período de seis semanas, houve a redução dos níveis de $\mathrm{AlC}$ e que $75 \%$ dos pacientes atingiram a meta da glicemia média semanal menor ou igual a $150 \mathrm{mg} / \mathrm{dL}$. Na literatura há alguns estudos comprovando a eficácia da intervenção in- tensiva com o uso da automonitorização glicêmica na melhora do controle glicêmico de pacientes diabéticos, porém não foi encontrado um programa de intervenção em tão curto espaço de tempo como o estudo atual (26-28).

Quanto ao consumo alimentar, a literatura mostra que há vários métodos para a avaliação do consumo, porém ainda não existe um "método ideal”, ou seja, isento de falhas, mas sim o mais adequado para determinada situação. $\mathrm{O}$ uso de inquérito alimentar para avaliar o consumo alimentar é suscetível a existência de sub-relato das porções alimentares. Este sub-relato pode ser de forma consciente, relatando quantidades menores do que as de fato consumidas, por constrangimento, ou até mesmo inconscientemente mediante a dificuldade em quantificar as porções de alimentos (29). Apesar dessa limitação, o uso de inquérito alimentar é aceitável, principalmente utilizando uma metodologia padronizada para coletar e analisar as informações, a fim de minimizar esses riscos, como foi realizado no presente estudo.

No atual estudo, optou-se pelo registro alimentar de três dias ( $\mathrm{R} 3$ dias), pois esse método reflete a dieta atual de um indivíduo ou grupo populacional e não se baseia na memória do participante, já que os alimentos são registrados, preferencialmente, durante o consumo e também podem ser registrados por um observador $(30,31)$. A fim de minimizar erros de medidas no inquérito alimentar, algumas técnicas no processo de coleta e análise dos dados dietéticos foram realizadas (32). Dessa 
forma, na coleta do registro alimentar, os pacientes recebiam informações prévias do preenchimento e um profissional nutricionista treinado conferia as informações registradas (31). Com relação à análise dos dados de consumo, houve um cuidado em obter um banco de dados dietéticos confiável, utilizando um software e tabelas de composição centesimal.

Os resultados encontrados neste estudo mostraram que os pacientes do grupo intervenção aderiram às condutas nutricionais propostas. Nota-se uma diferença estatisticamente significativa na ingestão de calorias, na porcentagem e na quantidade em gramas de lipídios e quantidade em gramas de carboidratos. A intervenção nutricional propôs um plano alimentar hipocalórico, com redução entre 500 e $1.000 \mathrm{kcal} /$ dia, a partir do gasto energético estimado para a maioria dos participantes desse grupo, uma vez que $92,9 \%$ dos pacientes desse grupo eram classificados como portadores de sobrepeso e obesidade no início do estudo. Com relação à distribuição dos macronutrientes, os valores médios apresentados pós-intervenção de seis semanas estavam de acordo com o preconizado $(2,7)$. Esse achado é de suma importância mediante as dificuldades dos pacientes em seguir as condutas nutricionais apresentadas por outros autores $(8,33)$.

A utilização do perfil glicêmico para a intervenção nutricional foi de grande valia para os resultados apresentados. Esse instrumento permite mostrar ao profissional bem como ao paciente a importância da adesão nutricional para o controle da glicemia, uma vez que hipo e hiperglicemias, quando ocasionadas por erros alimentares, podem ser corrigidas (24). Segundo a ADA, o uso da automonitorização permite ao paciente avaliar se as metas glicêmicas recomendadas estão sendo atingidas ou não, auxilia nos ajustes medicamentosos necessários e nas condutas não farmacológicas, tais como atividade física e intervenção nutricional (34). Durán e cols. sugerem que o perfil glicêmico faça parte da rotina e do tratamento do paciente diabético, até mesmo em indivíduos recém-diagnosticados (35).

Com relação ao consumo alimentar do grupo convencional, os resultados apontam para uma diminuição na quantidade de fibra. Os pacientes, no momento basal, apresentavam o consumo mínimo de $20 \mathrm{~g} /$ dia, porém esse valor se reduziu. O objetivo da conduta dietética dada a esse grupo era incentivar o consumo desse nutriente, a fim de manter hábitos alimentares adequados a esses pacientes. Porém, quando há uma orientação padrão convencional, não há uma avaliação prévia dos hábitos alimentares e a orientação pode ocasionar piora na composição da dieta. É provável que a orientação do consumo diário de frutas tenha ocasionado diminuição da ingestão de fibras. Assim, a recomendação dietética sem a participação da nutricionista teve aspecto negativo no consumo de fibra diário. Conforme demonstrado anteriormente, a avaliação do consumo alimentar é complexa e o nutricionista é o profissional mais capacitado para tal análise. Com base na pirâmide alimentar brasileira, o consumo de frutas pode ser de 3 a 5 porções ao dia, podendo ser alterado conforme a necessidade calórica individual e melhora da qualidade da alimentação. Contudo, essas porções devem estar inclusas no plano alimentar do paciente, sem prejudicar a quantidade total de carboidrato consumida $(2,36)$.

Sabe-se que a ingestão de fibra em quantidades suficientes apresenta um efeito favorável no metabolismo da glicose e da insulina (37). Portanto, há um consenso nas diretrizes em incentivar o consumo de alimentos fontes de fibras, tais como grãos integrais, leguminosas, hortaliças e frutas $(2,7,37)$. Em nosso estudo, os resultados mostraram uma correlação negativa entre a variação no consumo de fibra e a GMS, demonstrando um impacto positivo do aumento do consumo de fibras no controle glicêmico. De acordo com os cálculos, 9,5\% do controle glicêmico dependeram do consumo de fibras.

Apesar das condutas nutricionais do grupo intervenção visando a uma diminuição modesta na perda de peso, não foi observada diminuição estatisticamente significativa no IMC. Porém, esse índice não permite analisar alterações na composição corporal, ou seja, analisar variações no peso dependente da massa magra ou da gordura corporal. Assim, no nosso estudo, é possível que um aumento no conteúdo de água do organismo, dependente do melhor controle glicêmico, pudesse estar compensando a perda de gordura corporal induzida pelas modificações na dieta, justificando a manutenção do peso desses pacientes. Em contrapartida, houve redução da circunferência da cintura, que poderia estar refletindo uma pequena redução na gordura visceral.

Observando os dados da semana 12 , é possível verificar que as alterações na dieta e a melhora do controle glicêmico puderam ser mantidas. Concluímos, portanto, que a abordagem proposta pelo nosso grupo de educação em diabetes, visando a uma intervenção individualizada por meio de uma equipe multidisciplinar no que se refere à orientação nutricional, aos cuidados com a medicação e adequação do tratamento farmacológico, com base na determinação da glicemia média semanal, mostrou-se 
mais eficaz que a abordagem convencional para a obtenção do controle glicêmico de pacientes diabéticos do tipo 2 . Nossos resultados indicam que um consumo mais adequado de fibras na alimentação contribui para a obtenção de melhor controle da glicemia nesses pacientes. Análises posteriores são necessárias para avaliar a necessidade de reforço nas orientações nutricional e farmacológica para a manutenção do controle glicêmico no longo prazo.

Agradecimentos: ao Conselho Nacional de Desenvolvimento Científico e Tecnológico $(\mathrm{CNPq})$ pelo apoio financeiro.

Participantes do Grupo de Educação e Controle do Diabetes do Centro Integrado de Metabologia Cardiovascular do Hospital do Rim e Hipertensão e da Universidade Federal de São Paulo: Amanda Pires, Anita Sachs, Aparecida Fátima Bueno, Augusto Pimazoni Netto, Bianca Pires, Camila Costa, Clarissa Uezima, Ednir Apostólico, Elizabeth McKenzie, Erácliton de Souza, Fátima Regina da Silva, Fernanda Carvalho, Gabriela Cavicchioli, Graça Camara, Irma Cianfrone, Kátia Martins, Kátia Niglio, Mário da Silva Júnior, Marta Dahouni, Milena Alves, Moema Bueno, Mônica Gambá, Patrícia Fan, Patrícia Monteagudo, Patrícia Zach, Priscila Broinizi, Renê de Araújo, Regina Niglio, Sônia Couto e Tarcila Campos.

Declaração: para realização deste trabalho, contamos com o auxílio da Roche Diagnóstica, que contribuiu com a concessão de glicosímetros e fitas reagentes. Os autores declaram não haver conflitos de interesse científico neste estudo.

\section{REFERÊNCIAS}

1. American Diabetes Association. Clinical Practice Recommendations 2008. Diabetes Care. 2008;31 Suppl 1:S12-54.

2. Sociedade Brasileira de Diabetes [Internet]. Tratamento e acompanhamento do diabetes mellitus - Diretrizes da Sociedade Brasileira de Diabetes; 2007.

3. Mclellan KCP, Barbalho SM, Catallini M, Lerario AC. Diabetes mellitus do tipo 2, síndrome metabólica e modificação no estilo de vida. Rev Nutr. 2007;20(5):515-24.

4. Sartorelli DS, Franco LJ. Tendências do diabetes mellitus no Brasil: o papel da transição nutricional. Cad Saúde Pública. 2003;19 Suppl 1:S29-36.

5. Anderson JW, Randles KM, Kendall CWC, Jenkins DJA. Carbohydrate and fiber recommendations for individuals with diabetes: a quantitative assessment and meta analysis of the evidence. J Am Coll Nutr. 2004;23(1):5-17.

6. American Diabetes Association (ADA) and The European Association for the Study of diabetes (EASD). Management of Hyperglycemia in Type 2 Diabetes: A Consensus Algorithm for the Initiation and Adjustment of Therapy. A consensus statement from the American Diabetes Association (ADA) and the European Association for the Study of Diabetes (EASD). Diabetes Care. 2006;28(8):1963-72.

7. American Diabetes Association. Nutrition Recommendations and Interventions for Diabetes. A position statement of the American Diabetes Association. Diabetes Care. 2008;31 Suppl 1:S61-78.

8. Rivellese AA. Dietary habits in type II diabetes mellitus: how is adherence to dietary recommendations? Eur $\mathrm{J}$ Clin Nutr. 2008;62:660-4.
9. Internacional Diabetes Federation [Internet]. Global Guideline for Type 2 Diabetes Global Guideline forType 2 Diabetes. 1995. Disponível em: http://www.idf.org/Global_guideline. Acesso em: Mar 1, 2011.

10. Instituto of Medicine/Food and Nutrition Board. Dietary reference intakes for energy, carbohydrate, fiber, fat, fatty acids, cholesterol, protein and aminoacids (macronutrients). Washington: National Academy Press; 2002.

11. American Diabetes Association. Standards of medical care in diabetes. Diabetes Care. 2009; 32 Suppl 1:S13-61.

12. Recine $E$, Nascimento $M A B$, Ito MK. Guia alimentar para pessoas com diabetes tipo 2. Brasília: Ministério da Saúde; 2001.

13. Conselho Federal de Nutricionistas [Internet]. Prato Colorido Alimentação saudável. Disponível em: http://www.cfn.org.br/. Acesso em: Dez 14, 2008.

14. Pinheiro ABV, Lacerda EMA, Benzecry EH, Gomes MCS, Costa VM. Tabela para avaliação de consumo alimentar em medidas caseiras. São Paulo: Editora Atheneu; 2000.

15. Fisberg RM, Villar BS. Manual de receitas e medidas caseiras para cálculos de inquéritos alimentares. São Paulo: Sigmus; 2002.

16. Nut Win - Programa de apoio à nutrição [software]. Versão 2.5 for Windows. São Paulo: Departamento de Informática em Saúde da Universidade Federal de São Paulo; 2005.

17. Tabela Brasileira de Composição de Alimentos - TACO 2.0. Campinas: Núcleo de Estudos e Pesquisas em Alimentação, Universidade Estadual de Campinas (NEPA- Unicamp); 2006. p. 113.

18. United States Department of Agriculture (USDA). National Nutrient Database for Standard Reference, release 16. [base de dados na Internet]. Washington. Agricultural Research Service. Disponível em: http:// www.nal.usda.gov/fnic/foodcomp. Acesso em: Abril 2, 2009.

19. Tabela Brasileira de Composição de Alimentos - TBCA USP 5.0. [base de dados na Internet]. São Paulo. Departamento de Alimentos e Nutrição Experimental da Faculdade de Ciências Farmacêuticas USP e BRASILFOODS (Rede Brasileira de Dados de Composição de Alimentos). c1998 [atualizada em agosto de 2008. Disponível em: http://www.fcf.usp.br/tabela/. Acesso em: Abril 2, 2009.

20. Lohman TG, Roche AF, Martorell R, editors. Anthropometric standardization reference manual. Abridged edition. Champaign: Human Kinetics Books; 1991.

21. Frisancho AR. New standards of weight and body composition by frame size and height for assessment of nutritional status of adults and the elderly. Am J Clin Nutr. 1984;40:808-19. Disponível em: www.ajcn.org. Acesso em: Ago 20, 2008.

22. World Health Organization (WHO). Obesity: preventing and managing the global epidemic. Report of a WHO, Consultation on Obesity. Geneva; 1998.

23. Nathan DM. Translating the $\mathrm{A} 1 \mathrm{C}$ assay into estimated average glucose values. Diabetes Care. 2008;31(8):1473-8.

24. Internacional Diabetes Federation [Internet]. Guideline on Self-Monitoring of Blood Glucose in Non-Insulin Treated Type 2 Diabetes; 2009. Disponível em: http://www.idf.org/guidelines/smbg-t2d. Acesso em: Mar 20, 2011.

25. Makris K, Spanou L, Rambaouni-Antoneli A, Koniari K, Drakopoulos I, Rizos D, et al. Relationship between mean blood glucose and glycated haemoglobin in Type 2 diabetic patients. Diabetic Med. 2008;25(2):174-8.

26. Guerci $B$, Drouin $P$, Grangé $V$, Bougnères $P$, Fontaine $P$, Kerlan $V$, et al. Self-monitoring of blood glucose significantly improves metabolic control in patients with type 2 diabetes mellitus: the Auto-Surveillance Intervention Active (ASIA) study. Diabetes Metab. 2003;29:587-94.

27. Kempf K, Kruse J, Martin S. ROSSO-in-praxi: a self-monitoring of blood glucose-structured 12-week lifestyle intervention significantly improves glucometabolic control of patients with type 2 diabetes mellitus. Diabetes TechnolTher. 2010;12(7):547-53.

28. Polonsky WH. Structured self-monitoring of blood glucose significantly reduces A1C levels in poorly controlled, noninsulin-trea- 
ted type 2 diabetes. Results from the Structured Testing Program study. Diabetes Care. 2011;34(2):262-7.

29. Scagliusi FB, Lancha Jr AH. Subnotificação da ingestão energética na avaliação do consumo alimentar. Rev Nutr. 2003;16(4):471-81.

30. Fisberg RM, Martini LA, Slater B. Métodos de inquéritos alimentares. In: Fisberg RM, Slater B, Marchioni DML, Martini LA, editores. Inquéritos alimentares: métodos e bases científicos. $1^{\mathrm{a}}$ ed. Barueri: Manole; 2005. p. 2-29.

31. Buzzard M. 24-Hour dietary recall and food record methods. In: Willet WC, editor. Nutritional Epidemiology. 2nd ed. New York: Oxford University Press; 1998. p. 50-73.

32. Fisberg RM, Marchioni ML, Colucci ACA. Avaliação do consumo alimentar e da ingestão de nutrientes na prática clínica. Arq Bras Endocrinol Metab. 2009;53(5):617-24.

33. Thanopoulou A, Karamanos B, Angelico F, Assaad-Khalil S, Barbato $A$, Del Ben M, et al. Nutritional habits of subjects with type
2 diabetes mellitus in the Mediterranean Basin: comparison with the non-diabetic population and the dietary recommendations. Multi-Centre Study of the Mediterranean Group for the Study of Diabetes (MGSD). Diabetologia. 2004;47:367-76.

34. American Diabetes Association. Standards of medical care in diabetes - 2011. Diabetes Care. 2011;34 Suppl 1:S11-61.

35. Durán A, Martín P, Runkle I, Pérez N, Abad R, Fernández M, et al. Benefits of self-monitoring blood glucose in the management of new-onset Type 2 diabetes mellitus: the St Carlos Study, a prospective randomized clinic-based interventional study with parallel groups. J Diabetes. 2010;2(3):203-11.

36. Phillipi ST, Latterza AR, Cruz ATR, Ribeiro LC. Pirâmide alimentar adaptada: guia para escolha dos alimentos. Rev Nutr. 1999;12(1):65-80.

37. Mello V, Laaksonen DE. Fibras na dieta: tendências atuais e benefícios à saúde na síndrome metabólica e no diabetes melito tipo 2 . Arq Bras Endocrinol Metab. 2009;53(5):509-18. 